Patrizia Farinelli

\title{
Oltre il tempo attraverso il mito: Bontempelli e l'attualizzazione narrativa di un progetto teorico - da Eva ultima al Viaggio d'Europa
}

Parole chiave: Novecentismo, realismo magico, tempo, relativismo, destino, mito

\section{$1 \quad$ Linee di un rinnovamento artistico-culturale}

L'attenzione di Bontempelli per la natura e la funzione dell'arte e della letteratura è testimoniata da innumerevoli interventi di carattere saggistico e giornalistico oltre che dalla stessa opera letteraria, specie quella degli anni Venti, in cui la riflessione critica si elaborò in maniera privilegiata anche in forma metaletteraria. Mosso da un'esigenza costruttiva di rinnovamento, come teorico del Novecentismo egli traccia su »900«, Cahiers d'Europe et d'Italie (1926-1929), le linee di un progetto per una cultura che potesse essere al passo coi tempi ${ }^{1}$. E su quel progetto torna ripetutamente con precisazioni e integrazioni come rende conto L'avventura novecentista (1938), volume che raccoglie numerosi contributi di vario genere (articoli, lettere, note) fra cui quelli più rilevanti apparsi sui Cahiers $^{2}$.

Sentite inadeguate e fatte oggetto di critica e d'ironia sono sia le posizioni dei futuristi ${ }^{3}$, sia quelle di letterati difensori di letteratura intimista. Dall'arte Bontempelli pretende innanzitutto trasfigurazione ${ }^{4}$, capacità comunicativa, anonimità e autonomia rispetto al contesto di sviluppo. Tali caratteristiche non indicano, però, solo il profilo richiesto al prodotto artistico per il nuovo secolo: sono pensate pure, in termini sovraepocali, come proprie dei classici. Nella sua riflessione gli autori che continuano a tenere nel tempo hanno avuto la capacità di

1 La rivista era stata fondata nel 1926 assieme a Malaparte e i primi cinque numeri uscirono in francese. Già questa scelta indicava l'intenzione dei redattori di aprirvi un dibattito di respiro europeo.

2 Tra i numerosi studi sul progetto novecentista si vedano quelli di Airoldi Namer (1975), Saccone (1979), Buonanno (2003) e la monografia Piscopo (2001, 195-236).

3 La capacità di guardare senza pregiudizi alle arti applicate, ma anche il tono regolativo del discorso e un linguaggio spesso irriverente, avvicinano comunque anche il Bontempelli novecentista ai futuristi più di quanto egli fosse stato disposto ad ammettere.

4 In una nota su De Chirico scriveva: »[...] verità suprema dell'arte [...] è la trasfigurazione (non la 'deformazione' come molti s'ostinano a dire)« (Bontempelli, 2006, 22). 
dire l'universale dietro al fatto singolo, di svincolarsi dal contingente e di proiettarsi verso il nuovo ${ }^{5}$. Si precisa così il modo in cui egli concepisce la storia della letteratura, considerandone l'evoluzione non attraverso fattori extraletterari bensì su processi interni di rinnovamento, e si chiarisce altresì come intende la tradizione (»continuità intima, profonda, tra manifestazioni di inaspettata novità ( $\mathrm{AV}, 34$, corsivo nel testo). I testi assurti a classici rappresentano allora, dalla sua prospettiva, quei valori del sovratemporale, sovraspaziale, sovraindividuale che si augurava potessero imporsi come distintivi di un Novecento da lui ritenuto, alle soglie degli anni '20, non ancora veramente cominciato. Ed è nei classici che cerca esempi autorevoli di fondamento alla riflessione estetica.

Il realismo magico quale »tendenza « integrante dell'utopia culturale novecentista poggia sul rifiuto di un'arte concepita come mimesi. A seguirne le linee tracciate nei Cahiers il compito dello scrittore e dell'artista sarebbe di trasfigurare, attraverso l'attività immaginifica, situazioni date, tratte dalla realtà fenomenica, per mostrarne il doppio fondo. In questo senso l'arte è intesa come una sorta di magia ${ }^{6}$. Tale tendenza difende una visione del mondo e una poiesi artistica che mantengano alta l'esperienza dello stupore. Richiesta è quella capacità di uscire dal presente che sarebbe per Bontempelli il carattere basilare della poesia in senso lato (AV, 304 e 331). Non si era lontani, limitatamente a queste posizioni, da quanto sostenevano altri contemporanei: pensiamo in ambito italiano soprattutto a Savinio e alla sua difesa, qualche anno prima, su Valori plastici, di un'arte che ricercasse l'essenza dei fenomeni oltre il contingente e deformasse allo stesso tempo la verità emersa tramite l'ironia (Savinio, 2007, 45-63).

L'esigenza di una presa di distanza dall'immediato referente storico solleva la questione del rapporto tra letteratura e attualità che Bontempelli affronta, tra l'altro, in uno scritto di obiezione alle richieste rivolte al milieu culturale da funzionari dell'ambiente fascista. Vi sostiene che all'artista spetta rappresentare il proprio tempo in maniera indiretta $(\mathrm{AV}, 309)$. La richiesta di »inaderenza «, di distanza prospettica rispetto al presente (raggiungibile non da ultimo attraverso l'ironia) ${ }^{7}$ assieme alla necessità di uno sguardo nuovo sul mondo (da primitivi), sono dunque centrali entro il progetto novecentista (AV, 303-304).

5 Ancora in un articolo del '34 ribadiva: »[... l'arte ha per natura fondamentale di non ritrovare mai, di inventare continuamente la forma nuova « (Bontempelli, 1938, 331). Nel seguito L'avventura novecentista verrà indicata con $\mathrm{AV}$, mentre la sigla $\mathrm{OS}$ indicherà le Opere scelte).

6 Contini ridimensiona la portata che quel concetto assume nell'opera dello scrittore: »[...] la prétendue magie de Bontempelli a son origine dans la trouvaille d'une agudeza, d'une 'pointe'" (Contini, 1972, 280).

7 »L'ironia è la forma artistica del pudore al cospetto dei nostri sentimenti, è un modo di allontanarci dal contingente, di liberarci da un'aderenza troppo minuta con le superfici delle cose« (AV, 27). 
Altre richieste avanzate nei Cahiers concernono le strategie narrative: al soggettivismo romantico e tardo-romantico Bontempelli contrappone l'anonimità dissuadendo gli scrittori dal trasporre nell'opera delle esperienze personali. Similmente rifiuta un tipo di critica mirante a cercare nel testo i sentimenti dell'autore (AV, 32 e 92). La narrativa avrebbe dovuto lasciare spazio alla fantasia e all'inverosimile, incentrarsi sull'intreccio $(\mathrm{AV}, 28)$ e, in analogia ai miti classici, consistere di »favole e personaggi che possano correre il mondo«:

[...] noi cerchiamo l'arte d'inventare favole e persone talmente nuove e forti, da poterle far passare attraverso mille forme e mille stili mantenendo quella forza originaria; appunto come avvenne dei miti e dei personaggi delle due ere che ci hanno preceduto. $(\mathrm{AV}, 39)$

In altre parole Bontempelli mirava ad una letteratura alleggerita dal peso di istanze autorali: "[...] la vera e suprema forma poetica, direi che non è la esaltata descrizione del proprio stato d'animo (quale è di solito intesa la poesia lirica) ma appunto la creazione di miti« (AV, 169, corsivo nel testo). Tali richieste nascono dalla volontà di scrivere per il largo pubblico e in termini comunicativi. E proprio in vista di questi fini le opere della mitologia classica assurgono a esempi di cui tener conto ${ }^{8}$. In uno studio dettagliato sulle elaborazioni del mito nella letteratura italiana del ventesimo secolo Van den Bossche constata allora come nel progetto novecentista bontempelliano i richiami al mito abbiano due funzioni essenziali, quello di evidenziare, da una parte, una precisa richiesta narratologica (il mito vi appare: "mezzo collaudato per realizzare una narrativa rapida e dinamica«) e di chiarire, dall'altra, un'esigenza socio-culturale in quanto »il mito equivale ad un tipo di narrativa collettiva ed anonima 9 .

Sempre secondo quel progetto, che era cresciuto anche sull'influsso di letture nietzschiane, avrebbe dovuto imporsi un nuovo tipo d'individuo, non più soggetto disorientato, esposto al contingente e confuso con la massa, bensì cosciente del proprio destino e dei propri compiti. Sarebbe stato urgente, inoltre, perseguire una nuova temporalità e una nuova spazialità, capaci di fronteggiare le visioni soggettive tipicamente tardo-moderne di spazio e tempo.

Il còmpito più urgente e preciso del secolo ventesimo, sarà la ricostruzione del tempo e dello spazio [...]. Quando potremo credere di nuovo in un Tempo e in uno Spazio oggettivi e assoluti, che si allontanano dall'uomo verso l'infinito [...] potremo con sicurezza affrontare il secondo còmpito, che sarà il ritrovamento dell'individuo, sicuro di sè [SIC], sicuro d'essere sè, di essere sè e non altri, sè con alcune certezze e alcune responsabilità, con le sue

8 L'Odissea è definita un romanzo popolare. Cfr. AV, 298, 145 e 155.

9 Versione italiana dell'autore di un passo di »Justification« con cui si apriva il primo dei Cahiers. 
passioni particolari e una morale universale: e in cima a tutto ritroveremo forse un Dio, da pregare o da combattere. $(\mathrm{AV}, 17)^{10}$

È difficile pensare come un intellettuale assai lucido nel cogliere i fenomeni caratteristici di una società proiettata al veloce mutamento, i cui fondamenti ontologici ed epistemologici erano improntati alla coscienza del relativo, potesse pensare come radicabile nel 20. secolo il progetto di una civiltà basata su simili presupposti concettuali. L'arte da sempre ha a che fare con un soggetto 'altro' rispetto a quello contingente e da sempre persegue un tempo diverso da quello fenomenico. Bontempelli ne è era perfettamente consapevole: lo sottolineò parlando dei classici e lo rese esplicito nelle proprie opere. Il suo augurio in sede programmatica, come ben si può notare, non concerneva tuttavia solo l'ambito estetico; gli premeva invece partire da un rinnovamento dell'arte per ritrovare saldi assiomi che potessero valere anche in ambito ontologico ed etico. E tale fiducia lascia sconcertati.

Qui si cercherà di evidenziare come la teorizzazione del Novecentismo pesi sull'opera letteraria del suo propugnatore e ne freni l'effervescenza sperimentatrice, l'elemento ludico, ma anche la capacità di parlare in modo problematizzante e per nulla assertivo che caratterizzavano i suoi lavori nel decennio che va fin quasi alla fine degli anni Venti ${ }^{11}$.

\section{Dalla demistificazione alla costruzione}

In una postilla risalente al febbraio del 1929, ristampata nove anni dopo ne L'avventura novecentista Bontempelli scriveva:

Il diavolo ha bisogno che l'uomo non creda a sè stesso e al mondo [...]. Per qualche tempo la cosa gli è riuscita per mezzo del materialismo e poi del suo luogotenente il positivismo; quando non li ha più potuti tenere in piedi, ha dovuto ricorrere al relativismo [...]. La nostra dunque è un'impresa contro il diavolo. (AV, 46)

La preoccupazione che motiva tale impresa è quella dell'intellettuale del tardo moderno, ben cosciente del disorientamento concettuale indotto dalla fine di un pensiero basato sul dualismo oppositivo. Il teorico del Novecentismo non fa che condividere un disagio diffuso fra la stragrande maggioranza dei letterati della sua generazione e di quella immediatamente precedente. Tuttavia il ductus utopico con cui affronta il problema, le formule apodittiche che usa nell'argomentazione, la

10 Condivisibile la posizione di Baldacci (»Introduzione« a OS, XI-XLIII), secondo cui è alla fase del primo dopoguerra che risalgono le opere più riuscite dello scrittore.

11 Fontanella $(1983,142)$ parla al proposito di un »surrealismo razionalizzato«. 
consequenzialità con cui applica queste tesi nella produzione letteraria matura e più in generale la fiducia che lo porta a credere si possa mettere davvero riparo a quella tendenza di pensiero fanno di lui un caso isolato.

Nella prima metà degli anni Venti la sua operazione volta a contrastare il relativismo non sembra ancora iniziata. L'esigenza era piuttosto di farlo straripare, di portarlo all'assurdo e con una volontà enorme di sperimentare. Lo stile che impronta i lavori redatti tra il primo dopoguerra e il 1928 mostra un'originale elaborazione di imputs accolti dalle avanguardie ed esprime esigenze poetologiche condivise negli ambienti letterari più innovatori. Si pensi solo ai fenomeni di metalessi fra piano testuale ed extratestuale, all'inserzione come attanti, in più di un testo, di figure di manichini e burattini, o ancora alle situazioni surreali di alcuni racconti confluiti successivamente nella raccolta Miracoli. È appunto di quegli anni il Bontempelli che più si avvicina ai surrealisti, per quanto il suo surrealismo non abbia certo i tratti di quello teorizzato dai francesi risultando meno straniante oltre che privo di finalità politicamente eversive ${ }^{12}$.

Solo verso la fine di quel decennio si registra nel suo opus un decisivo cambiamento di poetica (non il primo ${ }^{13}$ ). Il rifiuto del relativismo si traduce anche in una revisione della figura del personaggio ${ }^{14}$. Nei suoi romanzi degli anni Trenta legati ai miti della modernità e basati su vicende che potevano nascere dalla cronaca, i personaggi non rappresentano allora più degli individui mossi dalla casualità: stanno per dei soggetti che adempiono il destino col senso di una scelta e combattono senza dubbi (da "eroi del bene o del male « contro un concetto contingente di tempo (AV, 265). Cambia di necessità la logica dell'azione e le vicende ricevono un conseguente epilogo. Se la scrittura bontempelliana riesce a quel punto meno convincente è anche perché perde quell'elemento d'irrisolto che, seppur dietro a soluzioni ludiche e grottesche, manteneva presente nei suoi testi l'inquietante.

\section{Due forme del dire: Eva ultima e Viaggio d'Europa}

A evidenziare il passaggio di poetica fra l'opera degli anni Venti e quella più tarda basterebbe il confronto fra due testi esemplari delle rispettive fasi di attività.

12 Gli scritti giovanili appartengono ad una fase ancora diversa di attività e vengono quasi interamente rifiutati.

13 Si rimanda per questo aspetto a un precedente articolo: Farinelli (2011).

14 Eva ultima (1923) fu ripubblicato nel 1940 assieme a La scacchiera davanti allo specchio nel volume Due favole metafisiche (1921-1922) in cui venivano introdotte due »Note a Eva ultima« contenenti dei testi pseudodocumentari miranti a dare uno statuto di realtà alla protagonista e a istituire una sovrapposizione tra l'autore empirico e il narratore. Tale strategia, che riportava la persona dell'autore dietro alla sua opera, avrebbe avuto la funzione di creare »une autobiographie fictive derrière laquelle il lui est loisible de se dissimuler« (Bouchard, 1997, 82). 
Scegliamo Eva ultima (1923) $)^{15}$ e Viaggio d'Europa $(1939)^{16}$ in quanto si prestano bene a un parallelo soprattutto per via di alcune analogie nella fabula.

In entrambi ha luogo una rivisitazione di miti antichi. Per Viaggio d'Europa Bontempelli rielabora quella che è per eccellenza una vicenda di rinascita, il mito della Fenice, e l'impianta sul mito di Europa sfruttando un elemento integrante di quest'ultimo che vuole appunto la Fenicia come patria della giovane. In Eva ultima (oltre a creare un gioco intertestuale col romanzo fantastico di Villers de l'Isle Adam, Evve future, del 1886) lancia qualche debole richiamo al mito di Persefone (OS, 357) ${ }^{17}$. E però, mentre nella favola metafisica i sotterranei riferimenti mitologici svolgono una funzione parodica e sarcastica ${ }^{18}$, in Viaggio d'Europa quell'operazione di ripresa (che ha un peso dominante sia per la struttura dell'azione sia per il senso del discorso in quanto la vicenda della Fenice struttura la riflessione sul tempo) si comprende solo a partire dalle posizioni espresse nei Cahiers sulle opere della mitologia classica come non trascurabili modelli di riferimento per una letteratura di marchio novecentista.

In Eva ultima la storia inizia con una profezia. Un'indovina annuncia a Eva, una donna con "molto passato e forse un po' di futuro«, che le sarebbe accaduta una "cosa nuova « in seguito alla quale sarebbe morta o rinata. Nell'episodio successivo appare, come dal nulla, una figura maschile in compagnia della quale la protagonista inizia un viaggio che la porta ${ }^{19}$, dopo un passaggio di soglia, in uno spazio inverosimile dove non riesce a fare i conti né con quell'Evandro che ve l'ha condotta, né con i personaggi che vi circolano, chiare caricature di cortigiani. Il dialogo fra Eva ed Evandro risulta impossibile; a bloccarlo sono le risposte sarcastiche e dissacranti di quest'ultimo. Eva è delusa sia dai modi di fare di Evandro, la cui presenza la isterilisce, sia dall'avventura in cui è venuta a trovarsi. Vorrebbe almeno del divertimento da questo "falso mago « $\mathrm{o}$ dio o demone, il quale fa apparire allora come per incanto Bululù, una marionetta con la facoltà di parlare e di obbedire. Ne resta affascinata, la interroga, constata in essa una condizione analoga alla sua, di prigioniera, e finisce per amarla e proteggerla. Come ne

15 Eva ultima (1923) fu ripubblicato nel 1940 assieme a La scacchiera davanti allo specchio nel volume Due favole metafisiche (1921-1922) in cui venivano introdotte due »Note a Eva ultima contenenti dei testi pseudodocumentari miranti a dare uno statuto di realtà alla protagonista e a istituire una sovrapposizione tra l'autore empirico e il narratore. Tale strategia, che riportava la persona dell'autore dietro alla sua opera, avrebbe avuto la funzione di creare "une autobiographie fictive derrière laquelle il lui est loisible de se dissimuler« (Bouchard, 1997, 82).

16 La stesura del racconto risale al 1939: fu pubblicato sul settimanale Tempo con altro titolo tra il 1939 e il 1940 e poi, nel 1941, nel volume Giro di sole.

17 Sulla ripresa, nel racconto, di questo mito riflette Langella (2008).

18 Benussi $(1999,566)$ fa notare che, a parte Viaggio d'Europa, nell'opus letterario di Bontempelli »[...] del mito classico vengono salvati solo alcuni frammenti, decontestualizzati e frantumati in sequenze introdotte in storie moderne."

19 Il viaggio si compie su un'automobile lussuosa, un oggetto che si impone quale marchio d'attualizzazione di una vicenda antica. 
La scacchiera davanti allo specchio (1922) sarà una situazione caotica, il degenerare di un convito, a far precipitare la situazione.

Tanto i motivi principali della favola (in primis il viaggio/ratto in un altro mondo), quanto i nomi dei protagonisti sono fortemente connotati, ma nell'economia del racconto, attraverso una ricontestualizzazione delle fonti, finiscono per perdere di allusività e allora contemporaneamente veicolano e disperdono il lettore nel processo ermeneutico. L'intero discorso si caratterizza del resto per frequenza di ambiguità, anacronie, contraddizioni, espressioni d'incertezza. Basti il seguente esempio:

Perché fu allora, sì, quando mi aggiravo così per la campagna, è stato questa mattina, quando lo ho incontrato. Certo. E forse visto per la prima volta. Mi ha parlato. Che cosa mi ha detto? Perché non ricordo? Forse è naturale ch'io non ricordi le sue parole. Tuttavia, ecco: a un certo punto ero convinta che io e Evandro ci conoscevamo da tempo; ma qui appare una contradizione, perché io possedevo due certezze: di conoscerlo allora per la prima volta, e insieme di conoscerlo da un pezzo; pure io allora non vedevo che fosse una contraddizione, ricordo che questa cosa non mi parve per nulla assurda. Non confonderti Eva. Forse il séguito fa tutto più chiaro. Quale séguito? (OS, 361-362, corsivo nostro) $)^{20}$

Le questioni centrali affrontate in Eva ultima circa il destino e la causalità dell'essere emergono entro un'indagine sulla natura delle "persone "suggerita dalla presenza stessa, nella storia, di una figura di marionetta ${ }^{21}$. Anche in questo caso la quête della protagonista ha la funzione di aprire delle questioni piuttosto che risolverle. Un solo esempio: "Dove sono? Non voglio saperlo. Non sei tu che mi ha portata qui: mi ci ha spinta... non so chi' « - osserva - ed Evandro la corregge: " Non si dice - non so chi -, si dice il destino.' « Eva prosegue: "'E ci rimango, come libera o come prigioniera? E se è un incanto, chi verrà a sciogliermi?' " Per tutta risposta si sente dire: »'Ruggero con l'anello di Melissa' " (OS, 368). Ma una cosa Eva comprende bene e la sconvolge: la più umana, la più spontanea e naturale creatura che abbia mai incontrato, quella con cui avverte di condividere un destino, è una marionetta.

Se le domande sull'origine e sulla causalità sono dominanti nel testo (e reggono dialoghi molto intensi), non vi resta marginale nemmeno la questione della temporalità. Non si pone tuttavia ancora come una riflessione sull'irreversibilità del tempo e sulla ricerca di una dimensione temporale assoluta, ma piuttosto come un tentativo di liberazione dal peso del passato. Già il solo titolo dell'opera, che rimanda in modo

20 Anche Fresu (2008, 26-27) analizzando il linguaggio di Bontempelli richiama l'attenzione (riferendosi tuttavia a Miracoli) sull'uso di formule esprimenti incertezza.

21 Invitata da Bululù a spiegare perché ci tenga a conoscere e capire le cose del mondo, Eva risponde: »Non le cose, le persone« (OS, 405). 
antitetico all'ironico romanzo fantastico di Villers de l'Isle Adam, Ève future $(1886)^{22}$ indirizza in tal senso. Necessita domandarsi quale preciso rinnovamento vada colto dietro al viaggio con funzione demistificante. Se si considera che il testo è fortemente basato sulla semantica dell'invenzione, creazione, falsificazione artistica, non potrebbe raccontare, questa favola metafisica, più che un rinnovamento umano (estraneo alla riflessione bontempelliana di quegli anni) l'utopia di un rinnovamento dell'arte e della letteratura verso una cultura consapevole che l'artefatto è più vero o altrettanto vero del preteso vero? In tal caso l'opera risentirebbe ancora di quell'intenzionalità che guidava i due 'antiromanzi' La vita intensa (1920) e La vita operosa (1921) ${ }^{23}$.

Nell'episodio conclusivo, in cui si narra del ritorno di Eva in un mondo verosimile, questa appare serena: »Era un ritorno, ma ella sapeva di non tornare verso il passato « (OS, 443). Difficile stabilire cosa motivi quella trasformazione. L'essere riuscita a lasciarsi alle spalle un passato che, attraverso un'esperienza singolare, le era apparso vuoto e pieno di disincanto oltre che basato su convinzioni e convenzioni che andavano radicalmente ripensate? Nonostante la fabula (tramite la profezia) dia per possibile un rinnovamento, lo stato di serenità in cui la protagonista appare nell'episodio conclusivo arriva a sorpresa. Tra l'inquietante avventura sapienziale nella dimensione spazio-temporale straniata e quel rientro solare in una realtà verosimile resta un vuoto logico-narrativo.

Nell' incipit il narratore affermava di non volere proporre una chiave interpretativa o piuttosto una morale della storia (»[...] spero che altri saprà cavarne ammaestramenti, la cui precisione a me sfugge « (OS, 343)) e anche in conclusione sarebbe tornato sulla necessità di sospendere ogni giudizio. Considerando però la funzione ludica e depistante dei passi metanarrativi bontempelliani, attivata fin dalla prima pagina del testo, simili precisazioni appaiono antifrastiche. Solo per evidenziare tale strategia si potrebbe ricordare che nel primo capitolo il narratore sostiene di aver "saputi da fonti dirette « i casi che narra per vanificare, però, subito dopo tale pretesa, tradizionalmente tesa a creare verosimiglianza, sottolineando l'irraggiungibilità dei luoghi in cui gli eventi sarebbero accaduti. Emerge qui ancora, dunque, quel ductus distruttivo sulle forme tràdite del narrare che aveva trovato il suo culmine ne La vita intensa. A esplicitare la rottura di attese di lettura collaborano negli altri capitoli le asserzioni del protagonista maschile. Rivolto ad Eva, questi afferma tra l'altro: "Tu aspettavi per lo meno un castello: rupi, burroni e poi stagni plumbei con dei salici, l'acqua che lambisce le muraglie ammuffite del maniero; una torma di presenze occulte; e qui, in

22 Il romanzo narra la creazione di un automa dalle sembianze femminili, provvisto d'intelligenza.

23 Le due opere (per un'analisi delle quali si rimanda a Mascia Galateria, 1977) smontano, attraverso la parodia, i meccanismi del romanzo ottocentesco e criticano le convenzioni borghesi. La prima delle due, prototipo del metaromanzo del Novecento, racconta - osserva Guglielmi $(1986,206)$ - il non poter raccontare. 
qualche atmosfera piena di fosforescenze e tremori, nascondere alla terra, chi sa? un supremo amore?’ «(OS, 367-368).

Mentre il narratore evita espliciti giudizi, il testo nel suo insieme sviluppa invece un discorso che riapre saperi dati per certi, rovescia concetti invalsi (quello di persona si sfaglia), segnala aporie concettuali, denuda l'essenza di ruoli e relazioni fra i sessi e, non da ultimo, ostenta la natura di artefatto dell'opera e ne vanifica le attese. La complessa struttura testuale e precise scelte retorico-discorsive cariche di allusioni, per un verso, ed elusive, per l'altro, vietano tuttavia un'ultima parola sul senso di tale discorso e lasciano l'opera aperta a plurime letture ${ }^{24}$.

Una storia di rinnovamento ritorna pure in Viaggio d'Europa (1939-1940) ${ }^{25}$ dove si sviluppa una riflessione sul significato di una temporalità segnata dalle tre dimensioni di passato, presente e futuro e si tematizza la ricerca di una dimensione che vi si sottragga. Il testo apre la trilogia di racconti Giro di Sole in cui ritornano singoli elementi di opere della tradizione ${ }^{26}$ e si affrontano questioni etiche (fra cui il telos dell'esistenza umana, gli scrupoli di coscienza, il rapporto fra singolo e comunità) a partire da vicende sollecitanti delle riflessioni sui concetti di tempo, individuo e spazio. I protagonisti rappresentano dei soggetti provvisti di forza interiore capaci di affrontare con sovranità il destino e di viverlo fino in fondo. Già col primo di questi racconti filtra nella scrittura bontempelliana una dimensione spirituale nuova, di un cristianesimo laico visto come un umanesimo rivoluzionario e come una dottrina della rigenerazione ${ }^{27}$.

Nella versione bontempelliana del mito, Europa ha potuto assistere alla rinascita della Fenice e cogliere »un annunzio» nello sguardo della leggendaria creatura. Ciò che vive in seguito - l'incontro col singolare toro e il passaggio in un altro spazio con momenti di fascino e disincanto ${ }^{28}$, ma soprattutto la scioccante presa di coscienza dell'irreversibilità del tempo - le offusca il ricordo di quell'evento. Constata a un certo

24 Assai differenti le letture critiche di quest'opera. Baldacci (1978) tenendo presente l'ipotesto francese ne evidenzia la riscrittura parodica, Fontanella (1992) vede nel viaggio narrato un percorso nell'interiorità, Micoli (1996) propone un'interpretazione incentrata sulla tipologia della figura femminile, Langella (2008) considera il testo come un discorso sul destino e lo legge in relazione al rinnovamento di miti classici, Giordano (2009) ne sviluppa una lettura metanarrativa.

25 Alle riscritture moderne di questo mito dedica attenzione Guthmüller (1993).

26 Vi si riprendono rispettivamente il mito antico, modalità del dialogo filosofico leopardiano »Dialogo di Cristoforo Colombo e di Pietro Gutierrez« e spunti narrativi dell'epica ariostesca.

27 In uno scritto del 1944 si legge: „Come al fondo di ogni rivoluzione trovi le parole di Cristo, così al fondo di ogni restaurazione trovi il Concilio di Trento (Bontempelli, 1946, 66).

28 Europa si trova a confrontarsi con la meschinità del dio che l'ha rapita e sedotta, il quale viene raffigurato come debole e impacciato di fronte a domande imbarazzanti («'Tu hai mai detto bugie'? 'Che c'entra?' Giove era molto imbarazzato. Gli era venuto detto - 'che c'entra?' ma capiva di dover dare una risposta migliore« (OS, 486). 
punto: »[...] ma intanto se tu ora tornassi indietro e mi rimettessi sul prato [...] io già non sarei più felice come allora, sarebbe oramai tutta un'altra cosa [...].«E ancora, sempre rivolta a chi l'ha rapita: "Non capisci, che anche se noi tornassimo come eravamo ieri, è l'oggi che non può tornare a essere l'ieri? «(OS, 470 e 487). Dopo aver ascoltato la profezia di Clori sul suo futuro di madre e di esule senza possibilità di ritorno (annuncio, quest'ultimo, che la fulmina), sgravata, sceglie di assumere come un dono la morte che sente vicina. La interpreta come adempimento alla promessa di rigenerazione colta nello sguardo della Fenice. Prima di morire comprende anche il motivo della punizione: l'essersi dimenticata per un lasso di tempo di quella promessa. È a quel punto che sogna la Fenice cui chiede se potrà rinascere e si sente rispondere: »[...] 'chi è nato una volta non potrà più, mai più, morire per sempre.' [...] 'Lo sforzo difficile di ogni vita è essere davvero vita e non una apparenza che non contiene niente'«(OS, 492).

Nonostante le analogie che il racconto ha, nella trama, con Eva ultima, ben diverso sviluppo vi assume la riflessione sul tempo e sul destino, come pure diverse sono le strategie retorico-discorsive che le configurano. Per quanto concerne queste ultime, scompare nel racconto, in modo analogo a quanto accade negli altri testi di Giro di sole, il discorso metanarrativo che in Eva ultima agiva in funzione ludica e straniante ed era un ulteriore generatore di complessità semantica. Tendono inoltre a scemare ambiguità lessicali, concettuali e d'azione ${ }^{29}$. La prosa appare visibilmente diversa da quella dei lavori del decennio precedente ${ }^{30}$ : perde la squadratura sintattica e lessicale per farsi più smussata, con una frequente aggettivazione tesa ad aggraziare o vivificare oggetti e situazioni (»acqua lontana cadente«, »odore prezioso«, »cenere tenera» (OS, 452453)). Rare risultano le rotture di tono che costituivano invece una delle caratteristiche peculiari della scrittura bontempelliana negli anni Venti e che si ritrovano in Eva ultima soprattutto nei dialoghi fra il personaggio maschile e femminile ${ }^{31}$. Quanto alla struttura narrativa, l'autore impartisce consequenzialità all'azione e inserisce nel racconto un logico epilogo: a motivare la raggiunta serenità della protagonista e a dare senso alla sua morte vengono le ragioni della Fenice ascoltate in sogno. In modo molto più netto di quanto non fosse in Eva ultima il destino della protagonista, voluto da un'istanza superiore, si fonde qui con la scelta consapevole di non vivere da morti.

29 Rispetto a Eva ultima, in Viaggio d'Europa il narratore tende a fornire indicazioni più sicure sulla vicenda. Elimina ogni ermetismo nella profezia. Nel riportare fatti e sensazioni toglie voce al personaggio.

30 Ricordiamo un giudizio autorevole: "Secco e decisamente grottesco ai suoi inizi, Bontempelli evolve a suo modo [...] verso una sorta di 'canto'" (Contini, 1988, 16).

31 Hanno luogo soprattutto come passaggi dalla spiritualità legata alla vicenda della Fenice alla prosaicità della figura di Giove. Si veda il passo in cui questi con insolenza afferma di aver »fame« (OS, 486). 
Il racconto visualizza la ricerca di un tempo sottratto appunto alla morte, che sembra coincidere con quello della spiritualità, e precisamente di una spiritualità cristiana. Come puntualizza Guthmüller (1993, 437): »Massimo Bontempelli cerca nel mito della principessa fenicia le origini non tanto dell'Europa classica (come Calasso) quanto di quella cristiana. « Molto concorre nel testo a questa rilettura. Potremmo osservare che in tale direzione va anche l'ironia sulla prosaicità di Giove. La risemantizzazione cui Bontempelli sottopone il mito classico non è di piccole proporzioni ${ }^{32}$; come ebbe a notare Dazzi $(1942,86)$, qui non si crede alla forza mitica degli dei, ma degli uomini e il racconto proporrebbe allora a giudizio di questo critico una moralità moderna. Il discorso sulla rigenerazione spirituale non resta, infatti, sovrastorico, ma si intreccia a quello di un preciso rinnovamento storico-culturale. Organizzato come augurio di qualcosa a venire, esso verte sul cambiamento segnato dall'avvento del cristianesimo. Accennando alla funzione storicamente svolta dall'Europa rispetto all'Oriente Bontempelli rilancia anche quello che doveva continuare a essere ai suoi occhi il compito culturale del vecchio continente. Nel testo si esprime dunque l'augurio che l'Europa dopo millenni di »tempesta (OS, 484) possa essere luogo di irradiazione di una nuova cultura ${ }^{33}$. Una simile posizione l'autore l'aveva già esposta anni prima, ma in quel preciso momento (il racconto fu redatto nel 1939) poteva essere suggerita anche da una preoccupazione politica non meglio esplicitata nel testo.

Pure Viaggio d'Europa si presta dunque a diverse letture: spirituale in primo luogo (la promessa salvifica e la tensione verso l'assoluto), filosofica (la ricerca della »quarta dimensione«) oltre che storico-culturale (il compito dell'Europa). Da questo punto di vista la scrittura bontempelliana non perde densità semantica. La differenza rispetto alla favola metafisica qui considerata sta piuttosto nel fatto che la parola assertiva diventa più marcata e l'intero discorso cerca coerenza logica. Il dire tende a farsi esortativo e talvolta sentenzioso acquistando una tonalità moraleggiante. Tanto più carico d'implicazioni filosofiche, esistenziali e poetologiche appare allora, al confronto, l'irrisolto domandare delle favole metafisiche, la parola sospesa o contraddittoria che le caratterizza ${ }^{34}$.

Il progetto ideologico-culturale bontempelliano di superamento del relativismo, specie se letto a posteriori tenendo presente gli sviluppi generali del pensiero e delle lettere dagli anni Trenta in poi in Occidente, appare una lotta persa in partenza, come se lo scrittore avesse sottovalutato la forza del »diavolo«. Il relativismo e con esso la

32 Benussi $(1999,566)$ richiama l'attenzione sull'atmosfera familiare in cui viene calata la vicenda.

33 Ne L'avventura novecentista si legge: »[...] l'epoca nostra è quella in cui si vorrà superare la contraddizione tra Oriente e Occidente $[\ldots] \lll(\mathrm{AV}, 103)$.

34 D'altro avviso Dazzi $(1942,85)$ che giudicò il racconto »[...] il capolavoro totale di Bontempelli, con l'accordo, veramente magico, anch'esso, dell'intelligenza, della sensibilità e della stessa eticità in una forma cristallina." 
crisi d'identità e la perdita di valore di salde categorie ontologiche non erano fenomeni arrestabili. Nelle intenzioni di questo intellettuale il luogo in cui agire avrebbe dovuto essere l'arte e le strategie da adottare in ambito letterario quelle di una narrativa quanto più possibile svincolata dal soggettivo e dal contingente. Tali intenti trovano non marginale attualizzazione nelle sue opere letterarie. Se nella fase del primo dopoguerra il Bontempelli scrittore aveva di mira soprattutto il rifiuto di istituzioni letterarie tramandate ed esortava a liberarsi dal peso del passato recente - esigenze espresse con una scrittura di gusto surreale, ambigua nelle formulazioni, esposta a contraddizioni e illogicità e non priva dell'elemento ludico - nel seguito persegue una narrativa capace di rispondere, piuttosto, alla ricerca di un concetto più saldo di tempo e di individuo; da qui storie incentrate su una lotta contro il divenire, il caso, la contingenza. Forse quelle intenzioni teoriche furono da lui seguite in modo troppo rigido perché si realizzasse »l'inaderenza « che trovava nei capolavori e che emerge nella sua produzione migliore. Per una Nemesi della storia o più correttamente per uno scollamento spesso esistente tra intentio ed attuazione di un'opera, le ragioni della letteratura teorizzate nel programma novecentista e il rispetto dell'autonomia dell'opera da ideologie e da norme prefissate appaiono meglio difese nel Bontempelli che persegue ancora un'attitudine antiletteraria. Tanto meno guidata da linee programmatiche, tanto più ricca, la sua scrittura letteraria, di suggestioni di pensiero.

\section{Fonti}

Bontempelli, M., L'avventura novecentista, Firenze 1938. (Nel testo sotto la sigla AV)

Bontempelli, M., Due favole metafisiche (1921-1922), Milano 1940.

Bontempelli, M., Dignità dell'uomo, Milano 1946.

Bontempelli, M., Opere scelte (cur. Baldacci, L.), Milano 19785. (Nel testo sotto la sigla OS)

Bontempelli, M., Realismo magico e altri scritti sull'arte (cur. Pontiggia, E.), Milano 2006.

Savinio, A., La nascita di Venere. Scritti sull'arte, Milano 2007.

\section{Studi}

Airoldi Namer, F., Gli scritti teorici di Massimo Bontempelli nei »Cahiers du 900« e la ricostruzione mitica della realtà, Studi novecenteschi 4, 12, 1975, pp. 248-269.

Benussi, C., Il mito classico nel riuso novecentesco. Marinetti, Savinio, Bontempelli, Gadda, Calvino, Humanitas n. s., 4, 1999, pp. 584-577.

Bouchard, F., Eva ultima, du roman à l'autobiographie fictive, Écritures autobiographiques, Les Cahiers de Paris VIII/Recherche, 1997, pp. 79-94. 
Buonanno, E., Il novecento immaginario di massimo Bontempelli, Studi novecenteschi 30, 66, 2003, pp. 239-262.

Contini, G., Altri esercizi, Torino 1972.

Contini, G. (cur.), Italia magica. Racconti surreali novecenteschi, Torino 1988.

Dazzi, M., Bontempelli narratore (o dell'avventura del magico), Urbino 1942.

Farinelli, P., Bontempellis Repositionierung des Subjekts aus der Perspektive des "Novecentismo", in: Das Subjekt in Literatur und Kunst (cur. Bartoli, S. et. al.), Tübingen 2011, pp. 147-162.

Fontanella, L., Il surrealismo italiano, Roma 1983.

Fresu, R., Tra specchi e manichini, La lingua »fantastica« di Bontempelli, Roma 2008.

Giordano, V., Dalle avventure ai miracoli. Massimo Bontempelli fra narrativa $e$ metanarrativa, London 2009.

Guglielmi, G., Un romanzo manifesto, in: Guglielmi G., La prosa italiana del Novecento. Umorismo, metafisica, grottesco, Torino 1996, pp. 198-210.

Guthmüller, B., Il mito di Giove ed Europa. Riflessioni sulle origini e il destino del nostro continente, Cenobio 42, 1993, pp. 435-442.

Langella, G., Riscritture del mito nell'Italia metafisica e surreale, in: Italia magica. Letteratura fantastica e surreale dell'Ottocento e del Novecento, Atti del convegno, Cagliari-Pula 7-10 giugno 2006 (cur. Caltagirone, G., Maxia, S.), Cagliari 2008, pp. 198-210.

Mascia Galateria, M., Tattica della sorpresa e romanzo comico di Massimo Bontempelli, Roma 1977.

Micoli, S., Bontempelli e la dissoluzione della femme fatale, Italica, 73, 1, 1996, pp. 44-65.

Piscopo, U., Massimo Bontempelli. Per una modernità dalle pareti lisce, Napoli 2001.

Saccone, A., Massimo Bontempelli, Il mito del '900, Napoli 1979.

Van de Bossche, B., Il mito nella letteratura italiana del Novecento: trasformazioni e elaborazioni, Firenze 2007. 
Patrizia Farinelli

\section{Onkraj meje časa skozi mit: Bontempelli in pripovedna aktualizacija teoretičnega projekta - od Eva ultima do Viaggio d'Europa}

Ključne besede: »Novecentismo«, magični realizem, čas, relativizem, usoda, mit

Bontempellijevo delo na področju pripovedništva, esejistike in publicistike zaznamujeta zavračanje relativizma ter zahteva po novih ontoloških in estetskih temeljih znotraj kulture, ki je pripravljena $v$ isti meri preseči tako poznoromantično kot tudi avantgardno izkušnjo. Med zahtevami sta umetnost in literatura, ki sta sposobni refleksije lastnega obdobja brez sprejemanja gole slučajnosti, pa tudi zahteva po delih, ki se sučejo okrog modernih mitov in ki jih ne zaznamuje avtorjeva subjektivnost, podobno kot je veljalo za klasična mitološka dela.

Bontempelli se $\mathrm{v}$ svojem pripovednem ustvarjanju otresa konvencij in neutemeljenih idej ter se med drugimi sredstvi zateka k literarnemu liku, ki je nagnjen $\mathrm{k}$ vpraševanju, tudi vpraševanju samega sebe. Takšen lik se pojavi tudi v delih Eva ultima in Viaggio d'Europa, v katerih se skozi podobno pripovedno strukturo (odtujitveno potovanje kot izkušnja spoznanja in skepse, ki v obeh primerih pripelje do preporoda) pokažeta jasna razlika diskurzivnih strategij in drugačna refleksija o času. V prvem primeru je skozi govor o položaju človeka obravnavana tudi potreba otresti se teže preteklosti in neutemeljenih konvencij, v drugem pa se kot osrednja tema pokaže iskanje četrte dimenzije, ki bi lahko razrešila nepovrnljivost časa. Primerjava obeh del pokaže odločilen poetološki preobrat: po intenzivni fazi del, ki so obravnavala in širila »novecentismo«, je Bontempellijev način govora od 30. let naprej bolj asertiven, namera po teoretiziranju pa njegovo pripoved obremeni in na bolj tog način utrdi besedilno sporočilo. 